\title{
Three-dimensional limit equilibrium analysis of slopes
}

\author{
R. H. CHEN and J.-L. CHAMEAU (1983). Géotechnique 33, No. 1, 31-40
}

\section{Professor J. N. Hutchinson and Dr S. K. Sarma, Imperial College of Science and Technology}

The Authors have addressed an important and rather neglected problem. Their treatment of it is insufficiently firm or comprehensive, however.

Most importantly, the conclusion that 'in certain circumstances the three-dimensional factor of safety obtained for cohesionless soils may be slightly less than that for the two-dimensional case' is questionable. The two-dimensional factors of safety for cross-sections within the ellipsoidal end sections will be higher than that for the central, critical two-dimensional crosssection. In addition, the intercolumn shear forces $\boldsymbol{R}$ contribute to the resisting forces. Therefore, on both these counts, the threedimensional factor of safety $F_{3}$ should be higher than $F_{2}$. To prove otherwise, the Authors would need to demonstrate that their result is not merely a function of the assumptions made in their analysis. Particularly for cohesionless soils, with $r_{\mathrm{u}}=0$, the critical slip surface is very shallow, end effects are minimal and the ratio $F_{3} / F_{2}$ will approach, but not fall bclow, unity.

Concerning the Authors' analysis, it appears from Fig. 3 that the factor of safety applied to the intercolumn shear forces is the same as the factor of safety on the basal shear surface. This is unlikely to be the case in practice. In addition, the assumption (p. 32) that the intercolumn shear stresses decrease linearly from a maximum value at the outermost point to zero on the centre line of the slide implies a rather unusual velocity distribution for the slide in plan. Furthermore, the vertical intercolumn shear forces $S$ in Fig. 2 are neglected in Figs 3 and 4 and in equations (9) and (10). In equations (5) and (6) it is not clear what value of $h$ should be taken. These expressions apply to $r_{\mathrm{u}}=0$ conditions: are they modified appropriately when the $r_{\mathrm{u}}=0.5$ case is considered?

It is reasonably assumed that the intercolumn forces in the $Z$ direction are a function of $K_{0}$. In the various analyses carried out, however, the value of $K_{0}$ used is not stated, nor is the considerable sensitivity of the ratio $F_{3} / F_{2}$ to the value of $K_{0}$ brought out, or even mentioned.
The three-dimensional failure surfaces used, composed of the most critical two-dimensional cylindrical slip surfaces with semi-ellipsoidal end surfaces added, are not the most critical threedimensional failure surfaces. This does not invalidate the comparisons made, but renders the later unqualified references to the 'critical circle' misleading. In this connection, the critical twodimensional failure surfaces for the Authors' first combination, $c^{\prime}=0, \phi^{\prime}=40^{\circ}, r_{\mathrm{u}}=0$ (p. 35), would be expected to be shallow and essentially parallel to the slope, and not as shown in Fig. 8.

The parameter $l_{\mathrm{s}} / \mathrm{H}$ is mathematically clear but is not easy to relate to actual landslides. The term $H$ is introduced (p. 35) without prior definition. It should have been shown, with proper cross-referencing, in Fig. 7.

The clarity of the paper is impaired by some drafting and typographical errors. For example, $l_{\mathrm{s}}$ appears to be defined differently in Figs 7 and 10, the axis of rotation in Fig. 7 does not coincide with the centre of the cylindrical slip surface shown, the toe of slip circle 1 in the upper diagram of Fig. 8 seems to have gone astray and, in Figs 9 and 10, the axes are not subdivided very accurately. In Fig. $10, \phi$ should be $15^{\circ}$ rather than $1.5^{\circ}$.

\section{Dr Å. Holmberg, Lund}

It is of interest that the solution of a similar problem was presented by the late Gösta Bjurström in Teknisk Tidskrift 1944, pp. 769771. He assumed the sliding body to be a solid segment of a sphere analogous to the cylinder in the two-dimensional sliding problem.

\section{Authors' reply}

Hutchinson and Sarma declare that the statement 'in certain circumstances the threedimensional factor of safety obtained for cohesionless soils may be slightly less than that for the two-dimensional case' is questionable. If a cohesionless soil column with unit thickness in the $Z$ direction is considered as shown in the paper as Fig. 1, the normal force $N_{3 D}$ depends both on $\alpha_{x y}$ and $\alpha_{y z}$ (Fig. 4 in the Paper). That is, without considering the side forces, $N_{3 \mathrm{D}}$ can 
be expressed as

$$
N_{3 D}=\frac{W}{\left(1+\tan ^{2} \alpha_{x y}+\tan ^{2} \alpha_{y z}\right)^{-0.5}}
$$

However, in the two-dimensional case, the normal force $N_{2 \mathrm{D}}$ is $\left(\alpha_{y z}=0\right)$

$$
N_{2 \mathrm{D}}=\frac{W}{\left(1+\tan ^{2} \alpha_{\mathrm{xy}}\right)^{-0.5}}
$$

The driving moments produced by the weight of the soil columns with unit thickness and the same height are the same in both cases. However, $N_{3 D}$ is less than $N_{2 D}$ and thus the resisting moment is smaller in the three-dimensional case. Hence, although the consideration of side forces will increase the three-dimensional factor of safety, it is still possible that $F_{3}$ may be less than $F_{2}$. The same conclusion may be found in the paper by Hovland (1977) and the discussion by Azzouz \& Baligh (1978). For cohesive soils, the resisting force is independent of the normal force, but depends on the bottom area of the soil column. The driving moments are the same in both the two-dimensional and the threedimensional cases, but the cohesive resistance is larger in the three-dimensional case and hence $F_{3}$ is greater than $F_{2}$ for all cases.

Although the factor of safety applied to the intercolumn forces is the same as the factor of safety on the basal shear surfaces, it does not mean that the failure will occur along the intercolumn surfaces. The intercolumn shear forces $R_{\mathrm{c}}$ and $\boldsymbol{R}_{\phi}$ are functions of the external forces $R_{\text {ext }}$ and $S_{\text {ext }}$ and of the distance from the central plane of the sliding mass. This assumption implies that the outermost point has the largest deformation and that there is no relative movement at the central plane.

The vertical intercolumn shear forces $S$ in Fig. 2 of the Paper have not been neglected. The results of intercolumn forces $R$ and $S$ (horizontal and vertical components respectively) are decomposed into cohesive and cohesionless parts, such as $R_{c} / F$ and $R_{\phi} / F$ in Fig. 3, $R_{c}$ and $R_{\phi}$ in Fig. 4, $R_{1}$ and $R_{2}$ in equations (9) and (10) of the Paper. These expressions are modified appropriately for the $r_{\mathrm{u}}=0.5$ condition.

$K_{0}$ was obtained from an empirical formula such as Jaky's equation. Since the parametric studies showed that the factors of safety are not very sensitive to the side forces assumed (in Table 1 of the Paper), the Authors did not intend to study the effects of $K_{0}$ on $F_{3} / F_{2}$.

The two-dimensional critical failure surface was searched by the computer program STABL2. The failure surfaces are assumed to be circular for all combinations of strength parameters of homogeneous slopes. For cohesionless slopes, the failure surfaces are shallower than those of slopes with other strength parameters.

The Authors apologize for several drafting errors

(a) in Fig. 10, $l_{\mathrm{s}}$ should be as defined in Fig. 7

(b) in Fig. 7, the axis of rotation should coincide with the centre of the cylindrical slip surface

(c) in Fig. 9, both the vertical and the horizontal axes are not divided accurately

(d) in Fig. 10, the distance on the vertical axis from 1.4 to 1.5 is shorter

(e) in Fig. 8, the toe of slip circle 1 is not drawn clearly

(f) in Fig. 10, $\phi$ should be $15^{\circ}$

(g) in Fig. $7, H$, the height of the slope, should be shown and defined on p. 35

(h) in Fig. 4, on the right-hand side, $R_{\alpha F 2}$ should be $R_{\mathrm{SF} 2}$.

Bjurström presented a simplified solution, making assumptions regarding the geometry (spherical failure surface) and the soil conditions (undrained behaviour with uniform shear strength). Furthermore, to the Author's understanding, he was essentially concerned with the stability of the foundation material below the embankment or slope.

The intention of the Authors was to propose a three-dimensional slope stability analysis which can handle rather complex geometry and soil conditions. They also presented a finite element computer program which can simulate the construction sequence of embankments.

\section{REFERENCES}

Azzouz, A. S. \& Baligh, M. M. (1978). Discussion on Three dimensional slope stability analysis method. J. Geotech. Engng Div. Am. Soc. Civ. Engrs 104, GT9, 1206-1208.

Hovland, H. J. (1977). Three dimensional slope stability analysis method. J. Geotech. Engng Div. Am. Soc. Civ. Engrs 103, GT9, 971-986. 